\title{
The Management of Accountability for Innovation in an Organization
}

\author{
Abu-Hussain Jamal ${ }^{1} \&$ Oleg Tilchin ${ }^{1}$ \\ ${ }^{1}$ Al-Qasemi Academic College of Education, Baqa El-Gharbieh, Israel \\ Correspondence: Oleg Tilchin, Computer Science Department, Al-Qasemi Academic College of Education, P.O. \\ Box 124, Baqa El-Gharbieh 30100, Israel.
}

Received: July 31, 2019

doi:10.5430/ijba.v10n5p65
Accepted: September 3, 2019

Online Published: September 17, 2019

URL: https://doi.org/10.5430/ijba.v10n5p65

\begin{abstract}
The suggested comprehensive three-step method for management of the employees' accountability for innovation is aimed at intensification of the innovation activity in an organization. The innovation process is characterized by suitability, feasibility, and applicability of the ideas. It is performed by the phases: finding new ideas, evaluation of ideas, development of ideas including their experimentation and implementation. Change of the innovation process characteristics causes the need of the accountability management. As a result of the management, the accountability characteristics such as a sphere, a level, and a measure of the employees' accountability for innovation are changed. The method is realized by sequence of the steps: setting accountability, evaluating accountability, and managing accountability. The steps are aligned with the innovative process phases. At the first step, the spheres and the levels of employees' accountability for generating ideas are set. At the second step, the spheres, levels, and measures of employees' accountability for development of the ideas are determined. The measure of accountability characterizes accountability of the members of the dynamic and heterogeneous group which is self-formed by employees as a result of the idea assessment. It is set equal to the idea value. The idea value is calculated by summation of assessments of the innovative process characteristics. At the third step, the spheres, levels, and measures of employees' accountability while development of the ideas are guided. Sharing accountability among the group members is based on their knowledge and skills. The preferable innovation direction and the key idea are revealed.
\end{abstract}

Keywords: innovation process, management of accountability

\section{Introduction}

An organization exists in a highly competitive environment. Survival and progress of the organization require continuous improvement of organizational performance. Prevailing factor of improvement of organizational performance is an innovative capability of an organization developed through employees' innovative activity (Lawson \& Samson, 2001). Hence, the management should create an organizational environment favoring the employees' innovative activity.

The employees' innovative activity is directed towards creation of innovations. An innovation can be determined as a result of applying knowledge and skills that promotes the creation of new processes, products, and services providing advantage of the organization in marketplace.

Creation of an innovation is caused by an innovation process (Jones, 2012). There are the various approaches to structuring and detailing the innovation process (Longdon, 2011). The innovation process comprising the phases: finding ideas, evaluation of ideas, and development of ideas that includes their experimentation and implementation is considered in this research.

Generating and proposing new ideas related with the key strategic directions of the organizational development are the objectives of the first phase. Evaluation of the ideas and comparison of their potential values concerning improvement of organizational performance are the objectives of the second phase. Development of ideas by providing alignment of opportunities determined during the evaluation phase with organizational capabilities are the objectives of the third phase.

The innovation process characteristics can be determined. These characteristics are suitability - conformity of an idea to the innovative direction meaning the extent of the idea contribution to realization of the direction, feasibility employees' ability to develop an idea, and applicability- possibility of an idea use. 
Successful performance of the innovation process can be attained by the management of innovation allowing the organization to respond to internal or external opportunities and influences through changes (Burns\& Stalker, 1994; Bettina von Stamm, 2008; Shephard \& Ahmed, 2010; Andriopoulos \& Dawson, 2014; Goffin \& Mitchell, 2017; Tidd \& Bessant, 2017). There are the following applicable mechanisms of an organizational culture which can be used for managing the innovation process: motivation, knowledge management (KM), and accountability.

Motivation of employees through appropriate reward system engenders and supports interest of employees to participate in creation and development of innovations and serves as an essential factor of innovation creativity inspiration (Nacinovic, Galetic \& Cavlek, 2009; Glasberg \& Ouerghemi, 2011).

The KM mechanism provides knowledge sharing among employees while the innovation process (Rodney, 2000; Botha \& Snyman, 2008; Dalker, 2011).

The accountability mechanism promotes the innovative activity through performance-driven accountability meaning personal accountability for the innovation process and its outcome that gives employees and an organization a sense of purpose (Dubnic \& Justice, 2004; Evans, 2008; Bregman, 2016). Creating efficient personal accountability should be based on desire and ability of an employee to participate in the innovation process.

The carefully designed accountability for innovation should provide complex use of all accountability characteristics. The accountability characteristics are a sphere, a level, and a measure of accountability. A sphere of accountability can be determined as a functional framework for the employees' accountability according to their roles in the innovation process. Three levels of accountability for innovation are distinguished. The first level focuses on personal accountability. The second level focuses on interpersonal accountability. The third level focuses on accountability of an organization. The measure of accountability serves as the quantitative assessment of employees' accountability for innovation.

The participants of the innovation process should hold accountability for the innovations. The inevitable change of the innovation process characteristics should induce the change of the accountability characteristics that allows to improve one. Because of that, the dynamic relationship between the innovation process and the employees' accountability is realized. Hence, the innovation process management should be aligned with the management of the employees' accountability for innovation.

The goal of this paper is to represent the comprehensive three step method of the management of the employees' accountability providing adjustment of the accountability to the innovation process and combined use of the aforementioned mechanisms for management of this process. The method can serve as the methodological basis for creation of the system of adaptive management of accountability for innovation in an organization.

\section{Related Research}

Research concerning the subject of this paper are examined by the directions: improvement of organizational performance through the innovation activity in an organization, managing innovation, and the management of accountability for innovation.

Improvement of organizational performance through innovation requires the practical actions based on suitable research of change and development of organizational processes including the innovation process. Poole et al. (2000) suggested criteria the research should satisfy. According to the criteria, exploration of change and development should combine all factors and mechanisms that influence these processes and use data extractive directly from the processes.

Tidd \& Bessant (2017) determined the requirements for building an innovative organization. These requirements are shared vision, leadership providing a creative climate, wide involvement in innovation activity, suitable organizational structure, key employees, effective teamwork, propelling knowledge, and supervising others.

Brands \& Kleinman (2010) offered the practical steps to achieve improvement of organizational performance and business growth through innovation. The basic steps are inspiring, creating needed values, building accountability, idea management, observing, measuring, and rewarding. Ettlie (2011) disclosed the dependence on innovation strategies from organizational strategies. The author described the innovation process and emphasized the important role of collaboration in this process.

Lawson \& Samson (2001) represented innovation management as a form of organizational capability. The authors determined a set of characteristics of an innovation capability of an organization. The set comprises vision and strategy, expertise and organizational intelligence, idea management, organizational structure, culture and climate, and technology management. 
Van de Ven (1986) determined factors affecting the management of innovation in an organization. The factors are new ideas, employees, interactions, and organizational environment. On the basis these factors the author formulated and considered the basic problems of managing innovation: a human problem, a problem in managing the innovation process, a structural problem, and a leadership problem. It allowed the author to propose framework to managing innovation.

Rodney (2000) established the role of knowledge management in maintaining and strengthening innovation in an organization. The author created KM model shaping integration of KM with the innovation process.

Evans (2008) affirmed improvement of organizational performance was resulted by embedding accountability into organization strategy, determining clear expectancies, and building relationships among group members on the basis of accountability. Olokundun et al. (2018) established workplace clarity provided positive essential influence on employees' accountability for innovation in an organization. Grenny (2014) explicated high performance of a collaborative group was provided by motivation and mutual accountability of group members.

The above review allows to conclude the dynamic relationship between the innovation process and the employees' accountability isn't disclosed. As a result of that, the employees' accountability hasn't been aligned with the innovation process. Hence, the challenges associated with the management of accountability for innovation in an organization remain undecided.

Furthermore, complex consideration of all accountability characteristics hasn't been represented; accountabilities for development of the ideas haven't been correlated with their values; a quantitative measure of the employees' accountability hasn't been introduced; knowledge and skills heterogeneous collaborative groups, the members of which share accountability for the innovation process and outcomes haven't been built; the collaborative group composition hasn't been adapted to changing the innovation process characteristics; sharing accountability among the collaborative group members on the basis of their knowledge and skills hasn't been provided; ordering of the innovation process hasn't been described; the accountability management coordinated with the determined order of development and implementation of the ideas hasn't been realized.

\section{Management of Accountability for Innovation in an Organization}

The need of management of accountability for innovation in an organization is engendered by change of the innovation process characteristics caused by a dynamic workplace. As appears from the above, these characteristics are suitability - conformity of an idea to the innovative direction reflecting important of an idea for the direction, feasibility - ability of an idea development, and applicability- possibility of an idea use. The innovation process is realized by the sequence of the following phases: finding new ideas, evaluation of ideas, and development of ideas.

The management of accountability is performed as follows. At the first, the current state innovation process is determined through assessment its characteristics. Then, the accountability management by taken into consideration the current state of the innovation process realizes change of the employees' accountability characteristics. According to the above, these characteristics are the sphere, the level, and the measure of accountability. Thus, adaptation of the accountability to the dynamic innovation process is provided. Changeable accountability of the employees allows to improve the innovation process. The accountability management process is presented by Figure1.

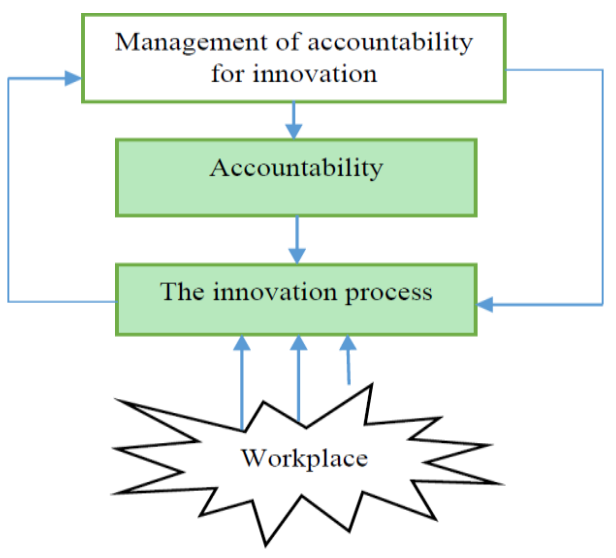

Figure 1. The accountability management process 
The suggested comprehensive three-step method of management of the accountability for innovation is aimed to adjust the dynamic relationship between the innovation process and the employees' accountability. Complexity of the method consists in combined use of the following mechanisms for management of an innovation process: accountability, motivation, and KM. The method provides setting, evaluating, and managing accountability of the employees for innovation. The method steps are aligned with the phases of the innovation process.

\subsection{The First Step: Setting Accountability}

The step objective is setting a sphere and a level of accountability of a leader, managers, and employees. This step is related to the innovation process phase "finding ideas".

At first, the leader's sphere of accountability on personal level of is set. The sphere includes determining the current goal of the organization to improve drastically organizational performance, revealing the innovative directions having the greatest potential of the innovative activity to attain the goal, and manifestation of the innovative directions in the organization.

Then, the managers' accountability sphere on personal level is set. It includes determining the employees' roles, creating conditions are aimed to provide their qualitative and motivated innovative activity, and building of an idea proposal format.

An employee's roles can be the following: an idea-creator, an idea-evaluator, and an idea-contributor. An idea-creator generates the idea and represents the idea for evaluation. An idea-evaluator assesses the proposed idea. An idea-contributor is an idea-evaluator who develops the idea together with an idea-creator.

The conditions promoting the innovation quality are the idea should be concerned with one innovative direction; the employee may be creator only one idea for the determined direction; the number of ideas that may be created by an employee for a few directions and the total quantity of ideas that may be offered by an employee are restricted.

The conditions promoting the employees' motivation are all employees participating in the innovation process are rewarded; size of the employee' reward depends on value of the idea and possible roles of the employee in the innovation process.

The idea proposal format serves as the frame for an employee's idea formulation. It includes description of objectives, originality, essence, suitability, feasibility, and applicability of the idea.

At last, the employee's sphere of accountability on personal level is set. It contains preparation of the idea proposal according to the format, and presentation of the idea proposal to colleagues for their familiarization. It stimulates innovation activity in an organization.

\subsection{The Second Step: Evaluating Accountability}

The step objective is evaluating accountability of the managers and the employees. This step is related to the innovation process phase "evaluation of the ideas".

The manager's sphere of accountability on personal level involves introduction of restrictions promoting qualitative assessment of the proposed ideas, and determination of the idea values.

The restrictions are the idea-creator may not be the valuator of peer ideas of the innovative direction to which his (her) idea belongs; the fixed number of the ideas may be assessed by the employee, the expert may assess the ideas belonging only one innovative direction; and the number of ideas for which the employee may be the contributor is limited.

The idea-valuator's sphere of the accountability on personal level consists in assessment of the proposed ideas. The employees and the experts may be the idea-valuators. The idea-creator's accountability sphere on personal level is creation and development of the idea. The idea-contributor' sphere of accountability on personal level consists in sharing accountability for development of the idea with the idea-creator.

Each proposed idea is assessed by the expert and the employees concerning the innovation process characteristics on the basis of the assessment range. The expert assessment is the more significant than the employee assessment. It is provided by introduction of the significant coefficient which may be different for the different innovation directions.

The idea value is determined by the manager as a result of summation of the expert and the employee assessments. The idea value reflects importance of the idea for improving organization performance and extent of success resulted by its realization. 


\section{Example 1:}

The assessment range is set from 1 to 10 . The significant coefficient of the expert assessment is equal to 3 . The idea assessments by the expert and three employees are represented by Table 1 .

Table 1. The idea assessments

\begin{tabular}{lllll}
\hline \multirow{2}{*}{$\begin{array}{l}\text { The innovation process } \\
\text { characteristics }\end{array}$} & The expert assessment & \multicolumn{3}{l}{ The employees' assessments } \\
\cline { 3 - 5 } & & The first & The second & The third \\
\hline Suitability & 8 & 5 & 6 & 7 \\
\hline Feasibility & 7 & 4 & 5 & 4 \\
\hline Applicability & 4 & 6 & 3 & 6 \\
\hline The idea assessments & $19 * 3=57$ & 15 & 14 & 17 \\
\hline
\end{tabular}

The assessments of the innovation by the expert and three employees are 57,15,14, and 17, accordingly. Then, the idea value is equal to 103 .

The sphere of accountability on interpersonal level of the idea-creator and the idea-contributors includes formation of collaborative groups, and sharing accountability for development of the ideas.

The employees assessing the idea may express desire to contribute in its development based on self-assessment of his or her ability to promote feasibility of the idea. Then, the idea-creator evaluates the possible contributors concerning compatibility by taking into account individual characteristics and availability knowledge and skills needed for development of the idea. As a result of this, a heterogeneous collaborative group is formed. The group contains compatible employees with mutual supplementation of knowledge and skills. The group members have interpersonal level of accountability.

The idea-creator and idea-contributors should take the most accountability for development of the idea which can have the most impact on improving organizational performance. It causes the need of correlation of the accountability measure with the result of the idea assessment. Hence, the measure of accountability of the collaborative group members for development of the idea is set equal to the idea value.

The idea-creator shares accountability for development of the idea with the idea-contributors. The accountability measure of the creator and the contributors can be determined by sharing the accountability measure pro rata the quantity of the differing knowledge and skills that they have for development of the idea.

Example 2:

The idea value is equal to 103 (Example1). Hence, the accountability measure of the collaborative group members is equal to 103. The collaborative group involves the idea-creator and two idea-contributors. The creator, the first contributor, and the second contributor have 5, 3, and 2 of differing knowledge and skills, accordingly. Then, the shared measures their accountability are 51.5, 30.9, and 20.6, accordingly.

\subsection{The Third Step: Managing Accountability}

The step objective is managing the employees' accountability for development of the ideas. This step is related to the innovation process phase "development of ideas".

The sphere accountability of the managers consists in determining the order of development of the proposed ideas. It assumes determining the sequence of the innovative directions according to their values, revealing the preferable innovative direction, and forming sequence of the ideas belonging this direction which should be firstly developed.

The sequence of the innovative directions is determined by their ordering in descending order regarding the values of the directions. The innovative direction value is determined by summation of the values of the proposed ideas related with the direction.

The preferable innovative direction is revealed by the choice from the sequence of the directions the direction having the most value. Thus, the preferable direction is the most important one for improving organization performance. If there are a few directions having the same and greatest value then the direction having the most suitability 
assessment is choose. If there are a few directions having equal assessments of suitability the direction with the most assessment of feasibility is choose. Thereby, the preferable direction is determined.

Example 3:

There two innovative direction. There are three proposed ideas regarding each direction. The ideas' primary assessments of the innovation process characteristics and the calculated idea values are represented by Table 2 .

Table 2. The primary assessments of the ideas

\begin{tabular}{|c|c|c|c|c|c|}
\hline \multirow{2}{*}{$\begin{array}{l}\text { Id innovative } \\
\text { direction }\end{array}$} & \multirow{2}{*}{$\begin{array}{l}\text { The idea } \\
\text { name }\end{array}$} & \multicolumn{3}{|c|}{ The primary assessments of the innovation process characteristics } & \multirow[t]{2}{*}{ The idea value } \\
\hline & & Suitability & Feasibility & Applicability & \\
\hline \multirow{3}{*}{1} & A & 40 & 36 & 25 & 101 \\
\hline & B & 42 & 40 & 26 & 108 \\
\hline & $\mathrm{C}$ & 38 & 35 & 23 & 96 \\
\hline \multirow{3}{*}{2} & $\mathrm{D}$ & 42 & 34 & 27 & 103 \\
\hline & $\mathrm{E}$ & 36 & 32 & 28 & 96 \\
\hline & $F$ & 42 & 34 & 30 & 106 \\
\hline
\end{tabular}

The value of each innovative direction is equal to 305 . It is calculated by summation of the idea values belonging the direction. It engenders the need of calculation of the suitability assessment for each direction by summation of the suitability assessments of the corresponding ideas. This procedure is performed for both directions. As a result of this, the suitability assessments of the directions is identical and equal to 120 .

Consequently, the feasibility assessments for the directions should be received. They are determined by summation of the feasibility assessments of the ideas related to the direction. As a result of calculation, the feasibility assessment for the first and second directions are 111, and 100, consequently. The feasibility assessment for the first direction is more than the feasibility assessment for the second direction. Owing to that, the first direction is the preferable direction.

Managing employees' accountability is caused by dynamics of the innovation process. The spheres, and levels of accountability are engendered by specificity of this phase of the innovation process. The measures of accountability of the managers, the creators, and the contributors of the ideas are correlated with changes of the innovation process characteristics while development of the proposed ideas. It causes the need to evaluate the changes and to affect to the innovative process through strengthened accountability.

The accountability sphere of the experts and valuators on personal level includes assessing change of the innovation process characteristics regarding the ideas belonging the preferable innovative direction. The idea for which the suitability assessment is increased can be determined as the key idea for further development and implementation since suitability means the extent of possibility of the idea to provide realization of the innovative direction. If there are a few ideas having the identical assessments of the suitability, the idea with the more assessment of feasibility is set as the key idea.

The accountability measure of the creator and the contributors of the key idea regarding the current state of its development is set equal to the current value of the key idea.

Example 4:

The preferable direction contains three ideas. The primary and current assessments of the innovation process characteristics for the ideas belonging this direction is represented by Table3. The primary assessments of the ideas are taken from Table 2. 
Table 3. The primary and current assessments of the ideas

\begin{tabular}{lllllll}
\hline $\begin{array}{l}\text { The innovation } \\
\text { process } \\
\text { characteristics }\end{array}$ & \multicolumn{2}{l}{ The idea A assessments } & \multicolumn{2}{l}{ The idea B assessments } & \multicolumn{2}{l}{ The idea C assessments } \\
\cline { 2 - 7 } & Primary & Current & Primary & Current & Primary & Current \\
\hline Suitability & 40 & 45 & 42 & 45 & 38 & 36 \\
\hline Feasibility & 36 & 34 & 40 & 38 & 35 & 32 \\
\hline Applicability & 25 & 23 & 26 & 25 & 23 & 20 \\
\hline
\end{tabular}

The current assessments of suitability for the ideas A and B are increased as compared with the primary assessments. These current assessments are identical and more than the current assessment of suitability for the idea $\mathrm{C}$. Hence, the current assessments of feasibility for the ideas A and B should be compared. The current assessment of feasibility for idea B is more than the current assessment of feasibility for idea A. Thus, the idea B is the key idea.

The current value of the key idea B is equal to 126 . Hence, the current measure of accountability of the creator and the contributors of the key idea is equal to 126 .

The sphere of accountability of the key idea-creator having personal level of accountability is increasing feasibility of the key idea if the current assessment of its feasibility is lower than the primary assessment. The creator transforms the primary collaborative group through involving in the group the contributors from the collaborative groups are formed for developing other ideas of the preferable direction. The transformation is based on compliance of the condition: the combined skills of the collaborative group members should be equal to feasibility-relevant skills (it is knowledge and skills needed for increasing feasibility of the key idea). The group members have interpersonal level of accountability for development of the key idea.

The sphere of accountability of the key idea-creator having personal level of accountability for increasing applicability of the key idea is determined as following. Composition of the collaborative group that was formed for increasing feasibility of the key idea is changed by inclusion of the contributors aimed at increasing applicability of the idea. It is achieved through compliance of the condition: the combined knowledge and skills of the collaborative group members should be equal to applicability-relevant knowledge and skills. The group members has interpersonal level of accountability for implementation of the key idea.

The sphere accountability of the managers is evaluating contribution of the key idea to the attainment of the objective of the preferable direction. If realization of the key idea is insufficient for attaining the objective then development and implementation of the next ideas are performed by similarly of the aforementioned way. It allows to determine the accountability measures of the creators and the contributors of the ideas concerning the current states of their development.

\section{Conclusion}

The suggested comprehensive three-step method realizing management of employees' accountability for innovation allows strengthening the innovation process that leads to improvement of organization performance. The accountability management is caused by the need of aligning accountability with the changeable characteristics innovation process reflecting its state.

Comprehensiveness of the method is expressed by realization of management by the all accountability characteristics and joint use of the organizational culture mechanisms such as accountability, motivation, and knowledge management providing complexity in management of the innovation process.

Particularity of the method consists in: adjustment of the dynamic relationship between the innovation process and the employees' accountability; adaptation of employees' accountability to changing the innovation process characteristics; correlation of the accountability measures for development of the ideas with their values that are changed while realization of the innovation process; self-formation of knowledge and skills heterogeneous collaborative groups accountable for the innovation process and outcome; adapting composition of a collaborative group to change of the innovation process characteristics; sharing accountability among the collaborative group members on the basis of their knowledge and skills; consistency of the accountability management with the determined order of development of the ideas.

The method can be applied as the practical tool of managing the employees' accountability for innovation in an organization. Further research will be directed to creation of the system of adaptive management of accountability for innovation. 


\section{References}

Andriopoulos, C., \& Dawson, P. (2014). Managing Change, Creativity and Innovation (2nd ed.). SAGE Publications Ltd.

Bettina, V. S. (2008). Managing Innovation. Design and Creativity (2nd ed.). Wiley.

Botha, A., Kourie, D., \& Snyman, R. (2008). Coping with Continuous Change in the Business Environment: Knowledge Management and Knowledge Management Technology. Chandice Publishing Ltd. https://doi.org/10.1533/9781780632056

Brands, R. F., \& Kleinman, M. J. (2010). Robert's Rules of Innovation: A 10-Step Program for Corporate Survival (1st ed.). Wiley.

Bregman, P. (2016). The right way to hold people accountable. Harvard Business Review. Retrived from https://hbr.org/2016/01/the-right-way-to-hold-people-accountable

Burns, T., \& Stalker, G. M. (1994). The Management of Innovation. Oxford University Press. https://doi.org/10.1093/acprof:oso/9780198288787.001.0001

Dalker, K. (2011). Knowledge Management in Theory and Practice (2nd ed.). The MIT Press.

Dubnic, J., \& Justice, J. B. (2004). Accounting for Accountability. Annual Meeting of the American Political Science Association. Retrived from http://mjdubnick.dubnick.net/papersrw/2004/dubjusacctg2004.pdf

Ettlie, J. (2011). Managing Innovation (2nd ed.). Taylor \& Francis.

Evans, H. J. (2008). Winning with Accountability: The Secret Language of High-Performing Organizations. Corner Stone Leadership Institute.

Glasberg, R., \& Ouerghemi, K. (2011). Innovation in Human Resources: A Theoretical Advancement on Employee Motivation and Organizational Innovation. $2^{\text {nd }}$ International Conference on Economics, Business and Management. IPEDR, 22. IACSIT Press, Singapore.

Goffin, K., \& Mitchell, R. (2017). Innovation management: effective strategy and implementation (3rd ed.). Red Globe Press.

Grenny, J. (2014). The best teams hold themselves accountable. Harvard Business Review. Retrived from https://hbr.org/2014/05/the-best-teams-hold-themselves-accountable?referral=03759\&cm_vc=rr_item_page.bott om

Jones, T. (2012). On Innovation. Essential Ideas, Inc.

Lawson, B., \& Samson, D. (2001). Developing Innovation Capability in Organizations: A Dynamic Capabilities Approach. International Journal of Innovation Management, 5(3), 377-400. https://doi.org/10.1142/S1363919601000427

Longdon, M. (2011). The innovation Master Plan: The CEO's guide to innovation. Innovation Academy. Walnut Greek, CA.

Nacinovic, N., Galetic, L., \& Cavlek, N. (2009). Corporate culture and innovation: implications for reward systems. World Academy of Science, Engineering and Technology, 397-402. Retrived from http://www.waset.org/publications/8755

Poole, M. S., Van de Ven, A. H., Dooley, K., \& Holmes, M. E. (2000). Organizational Change and Innovation Processes: Theory and Methods for Research. Oxford University Press, USA

Olokundun, M. (2018). Work transparency and innovation accountability in a selected organization in Nigeria. Academy of Entrepreneurship Journal, 24(4), 1-6.

Rodney, M. A. (2000). Knowledge Management as a Catalyst for Innovation within Organizations: A Qualitative $\begin{array}{llll}\text { Study. } \quad K n o w l e d g e & \text { and }\end{array}$ https://doi.org/10.1002/1099-1441(200010/12)7:4<233::AID-KPM94>3.0.CO;2-F

Shephard, P., \& Ahmed, C. (2010). Innovation Management. Pearson.

Tidd, J., \& Bessant, J. (2017). Managing Innovation: Integrating Technological (6th ed.). Market and Organizational Change, Wiley.

Van, De V., \& Andrew, H. (1986). Central Problems in the Management of Innovation. Management Science, 32(5), 590-607. https://doi.org/10.1287/mnsc.32.5.590 